\section{$\mathrm{X}$ (and G) mark the spot}

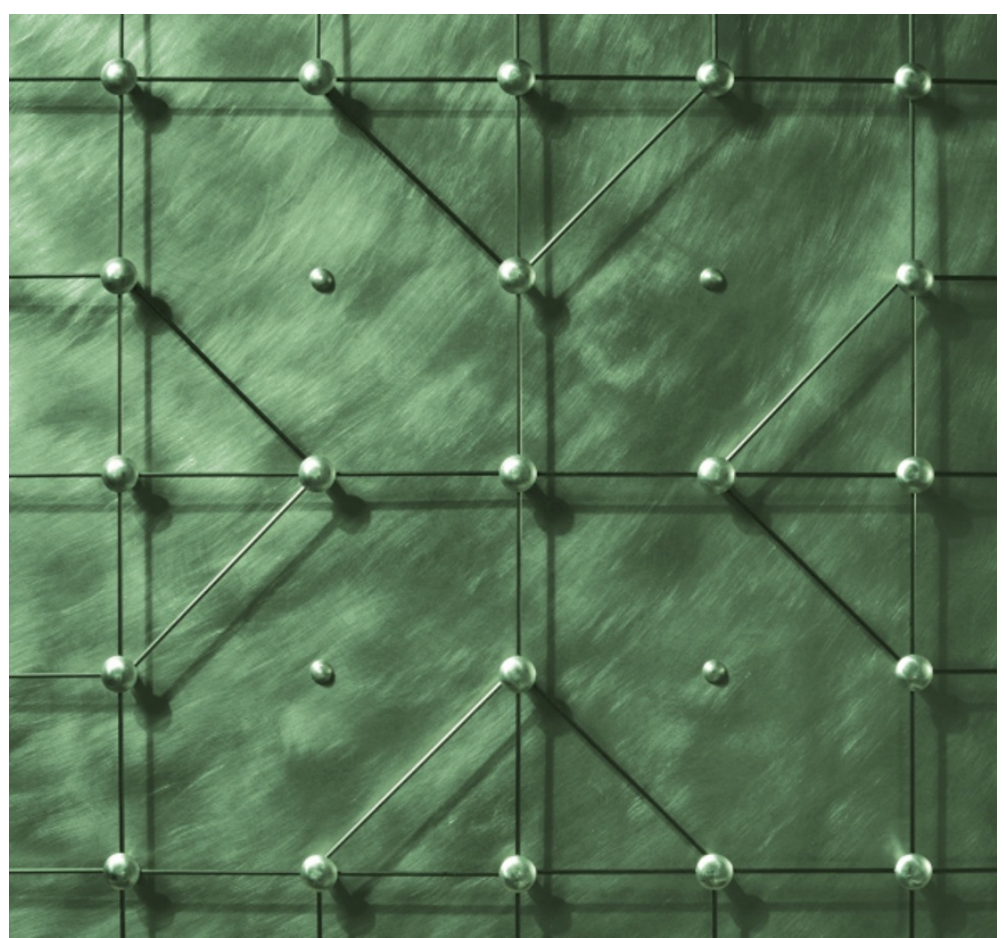

Fragile $\mathrm{X}$ syndrome is a genetic disorder that is characterized by mental retardation, enlarged testicles and mild craniofacial malformations. It results from defects in the fragile $\mathrm{X}$ mental retardation protein (FMRP), an mRNA-binding protein that is incorporated into messenger ribonucleoprotein particles (mRNPs) and can associate with translating polyribosomes. A working model to explain fragile $\mathrm{X}$ syndrome is that defects in the protein alter its ability to bind target mRNAs, misregulating their translation and leading somehow to the mental and physical features of the disease. But what are the mRNAs that FMRP can bind? Two recent papers published in Cell have taken independent routes to address this crucial question, and have neatly converged on the same answer.

Brown et al. immunoprecipitated FMRP-containing mRNPs isolated from mouse brains, probed microarrays with the co-purified mRNAs, and identified 432 transcripts that could potentially bind to FMRP. In a parallel experiment, the authors interrogated microarrays with mRNAs from polyribosomes obtained from patients with fragile $\mathrm{X}$ syndrome, looking for mRNAs with abnormal profiles as a result of FMRP deficiency. They identified 251 transcripts and showed that these mRNAs included many human orthologues of the mouse mRNAs co-purified in their first experiment.

Darnell et al. used an in vitro RNA selection assay to identify the sequence recognized by FMRP. They tested the ability of FMRP to interact with RNAs from a random pool, and carefully selected for sequences that could bind the protein. The authors succeeded in identifying a sequence known as G quartet, and established that it is necessary for the interaction between RNA and FMRP. Darnell et al. then looked for putative FMRP-interacting mRNAs in databases, and went on to show that some of the predicted transcripts did indeed bind to FMRP. Moreover, they also found that some of the mRNAs identified by Brown et al. could

\title{
Putting the MHB in its place
}

In vertebrates, the mid-hindbrain boundary (MHB) provides essential organizing signals for the patterning of the midbrain and rostral hindbrain. We know that the MHB forms where the caudal boundary of Otx2 gene expression meets the rostral boundary of $G b x 2$ expression. However, it is still unclear exactly how these and other factors interact to generate and position the MHB, and to activate MHB-specific genes such as $\mathrm{Fg} f 8$, which is also important for its signalling activity. Three different teams have addressed these questions, and have all presented their findings in recent papers, two published in Development and one in Nature Neuroscience.

It was shown previously that inactivation of either $O t x 2$ or $G b x 2$ does not prevent the formation of the MHB, but does alter its position. So, loss of Otx 2 shifts the MHB rostrally, whereas loss of $G b x 2$ shifts it caudally. In each case, there is a corresponding shift in the position of the Fgf8 expression domain, so that its rostral boundary remains at the MHB. From these observations, it was inferred that neither $O t \times 2$ nor $G b x 2$ was required to activate Fgf8. However, it could equally be argued that either gene alone was sufficient.

Now, Martinez-Barbera et al. and Li and Joyner have revisited this problem by examining the effects of inactivating Otx2 and $G b \times 2$ simultaneously in mice. They showed that $F g f 8$ expression does not depend on the function of Otx2 and Gbx2, because it was still activated in the double mutants, although its expression domain was broader, extending throughout the anterior neural plate.

If $F g f 8$ can still be expressed in the absence of both Otx2 and Gbx2, which factors are responsible for its initial activation? Using a gain-of-function approach in the chick embryo, Ye et al. showed that Pax 2 can activate $F g f 8$, although once the MHB has formed, Otx2 and Gbx2 seem to be required to maintain and refine its expression domain.
So, the induction and positioning of the MHB seem to depend on the integration of different molecular mechanisms. Pax 2 is sufficient to activate MHB-specific genes such as $F g f 8$, but $O t x 2$ and $G b x 2$ are both required to determine the final positions of their expression domains. Future studies should help to clarify the nature of the interactions between these and other factors, and to identify the source of the signals that activate Pax2 in order to initiate the whole process. Heather Wood

(2) References and links ORIGINAL RESEARCH PAPER Ye, W. et al. Distinct regulators control the expression of the mid-hindbrain organizer signal FGF8. Nature Neurosci. 4, 1175-1181 (2001) | Martinez-Barbera, J. P. et al. Regionalisation of anterior neuroectoderm and its competence in responding to forebrain and midbrain inducing activities depend on mutual antagonism between OTX2 and GBX2. Development 128, 4789-4800 (2001) | Li, J. Y. H. \& Joyner, A. L. Ot×2 and Gbx2 are required for refinement and not induction of mid-hindbrain gene expression. Development 128, 4979-4991 (2001)

FURTHER READING Wurst, W. \& Bally-Cuif, L. Neural plate patterning: upstream and downstream of the isthmic organizer. Nature Rev. Neurosci. 2, 99-108 (2001) 
bind to FMRP; not surprisingly, most of the transcripts identified in the microarray experiments contained a $\mathrm{G}$ quartet.

What are the proteins encoded by the mRNAs identified in the two studies? A significant fraction of them, molecules such as semaphorin $3 \mathrm{~A}$, potassium channel subunit Kv3.1 and microtubule-associated protein $1 \mathrm{~B}$, participate in different aspects of synaptic physiology and development. And although it would be premature to attempt to establish a link between these proteins and the characteristics of fragile $\mathrm{X}$ syndrome, their identification is certainly a significant step towards understanding the most frequent form of mental retardation.

Juan Carlos López

(2) References and links ORIGINAL ReSEARCh PAPERS Brown et al. Microarray identification of FMRP-associated brain mRNAs and altered mRNA translational profiles in fragile X syndrome. Cell 107, 477-487 (2001) fragile X syndrome. Cell 107, 477-487 (2001) |
Darnell, J. C. et al. Fragile X mental retardation protein targets $\mathrm{G}$ quartet mRNAs important for neuronal function. Cell 107, 489-499 (2001) FURTHER READING Chelly, J. \& Mandel, J-L. Monogenic Rauses of Xlinked me Nature Rev. Genet. 2, 669-680 (2001)

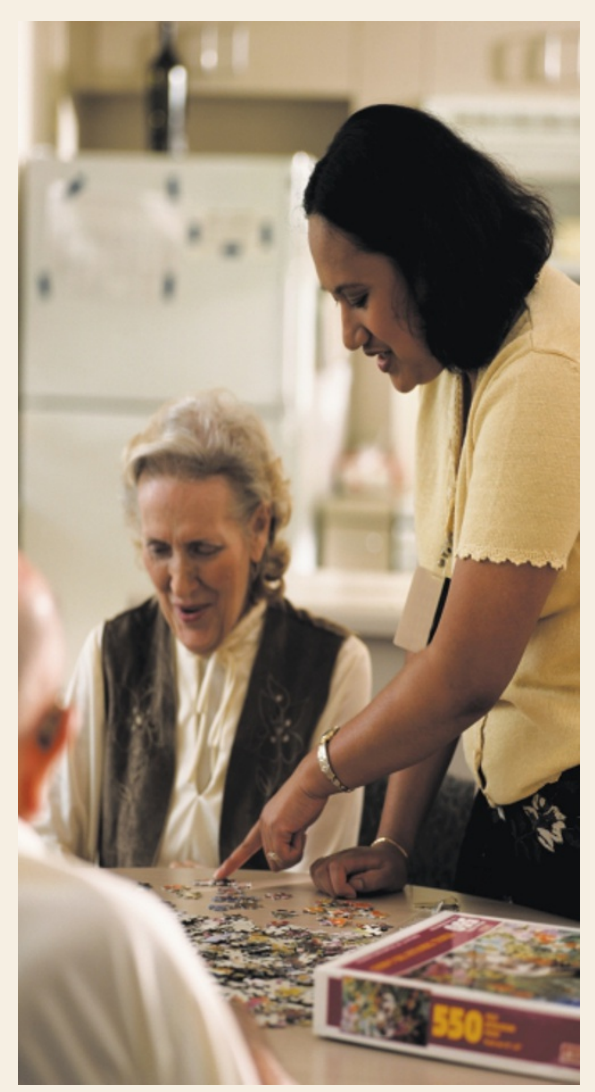

FUNCTIONAL IMAGING

\section{Monkey see, fMRI do}

Much of what we know about the primate visual system has come from single-cell recordings in the macaque monkey. These experiments have complemented functional imaging studies in humans, but it has been impossible in most cases to make direct comparisons between monkey and human brains, because we could neither use dynamic whole-brain functional imaging in the monkey nor carry out single-cell recording in healthy humans.

However, recent developments have made it possible to apply functional magnetic resonance imaging (fMRI) to monkeys. Until now, the best results have been obtained by using very high magnetic fields, much higher than those produced by the 1.5 Tesla scanners used in most human studies, and by scanning anaesthetized monkeys. But a new study by Vanduffel $e t$ al. has shown that excellent results can be obtained with a 1.5 Tesla scanner and awake monkeys, if an MRI contrast agent is used, rather than scanning for the blood oxygen level dependent (BOLD) signal that is often used in human studies.

Vanduffel and colleagues injected their monkeys with monocrystalline iron oxide nanoparticles (MION) before the experiments. MION have a long half-life in the blood, and can be detected by fMRI because increased local cerebral blood volume in a particular area of the brain causes an increase in the local concentration of iron oxide. This contrasts with the BOLD signal, which results from a decrease in the concentration of deoxyhaemoglobin as venous blood becomes more oxygenated in areas of increased blood flow and volume.

MION gave an approximately fivefold increase in sensitivity compared with BOLD. The authors comment that the application of MION fMRI to awake monkeys (which were trained to sit forwards in a restraining chair and look at a visual display during scanning) was "surprisingly straightforward", and that it also gave better spatial resolution and statistical power than the BOLD technique. Certainly, the use of MION has great potential for allowing us to compare patterns of brain activation directly between humans and monkeys. But what did Vanduffel and colleagues find in this study?

The focus of their investigation was the response of the monkey visual system to moving stimuli. We know from single-cell recordings that certain areas of the monkey brain contain neurons that respond to stimuli that are moving in a particular direction, and it has been proposed that these areas belong to a 'dorsal processing stream'

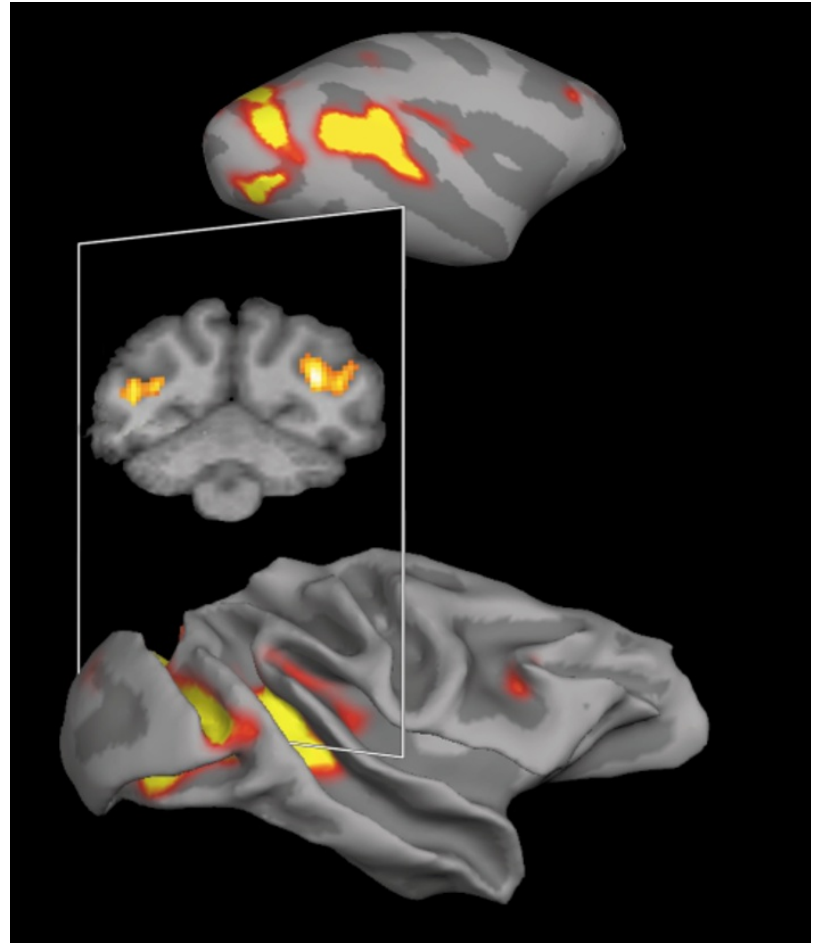

that is specialized for processing visual motion. In humans, fMRI has shown that many of the presumptive homologous areas are activated by moving stimuli. Vanduffel et al. found that those areas in the monkey brain that contained motionsensitive neurons were, as predicted, activated by moving dots or lines. But they also found that some other areas - such as area V4, TEO and parts of the intraparietal sulcus - were activated by moving lines (but not dots), despite not having been implicated previously in motion processing in monkeys. These areas had been shown to be activated by moving stimuli in humans.

The data seem to confirm that many cortical areas are functionally equivalent between monkeys and humans. In future, the use of MION fMRI should allow functional imaging to be more widely applied to awake monkeys. At last, we will be able to compare brain activation in monkeys with that in humans; and that might allow us to draw conclusions about whether the neuronal activity measured by single-cell recordings in monkeys can be extrapolated to humans.

Rachel Jones

(2) References and links

ORIGINAL RESEARCH PAPER Vanduffel, W. et al. Visual motion processing investigated using contrast agent-enhanced fMRl in awake behaving monkeys. Neuron 32, 565-577 (2001)

ENCYCLOPEDIA OF LIFE SCIENCES brain imaging: localization of brain functions | magnetic resonance imaging 